\title{
Liver Fibrosis and Steatosis in Virally Suppressed HIV- Infected Patients with Cytomegalovirus Seropositivity
}

\author{
Chyntia Olivia Maurine Jasirwan,**, Adik Wibowo*, Amal C Sjaaf*, Gita Aprilicia ${ }^{* *}$ \\ Dyah Purnamasari***, Evy Yunihastuti****, Rino Alvani Gani** \\ *Magister of Hospital Administration, Department of Health Policy Administration, Faculty of Public \\ Health, Universitas Indonesia, Depok \\ ${ }^{* *}$ Division of Hepatobiliary, Department of Internal Medicine,Faculty of Medicine, Universitas \\ Indonesia/Dr. Cipto Mangunkusumo National General Hospital, Jakarta \\ ${ }^{* * *}$ Division of Endocrine and Metabolic, Department of Internal Medicine, Faculty of Medicine \\ Universitas Indonesia/Dr. Cipto Mangunkusumo National General Hospital, Jakarta \\ ****Division of Allergy and Immunology, Department of Internal Medicine, Faculty of Medicine \\ Universitas Indonesia/Dr. Cipto Mangunkusumo National General Hospital, Jakarta
}

\section{Corresponding author:}

Chyntia Olivia Maurine Jasirwan. Division of Hepatobiliary, Department of Internal Medicine, Dr Cipto Mangunkusumo National General Hospital. Jl. Diponegoro No.71 Jakarta Indonesia. Phone: +62-21-31900924; facsimile:+62-21-3918842.E-mail: chynmadu@gmail.com; hepatologybilierfkuirscm@gmail.com

\begin{abstract}
Background: Cytomegalovirus (CMV) is a human herpesvirus common in people with human immunodeficiency virus (HIV). In a patient with immunocompetence, long periodic asymptomatic CMV might affect to develop the abnormal liver function and contribute to non-AIDS defining morbidity, including chronic liver disease. This study aims to know the prevalence of liver fibrosis and steatosis in virally suppressed HIV infected patients with CMV reactive and summarize the correlation of clinical presentation with liver fibrosis and steatosis in these subjects.

Method: A cross-sectional study in HIV Integrated Care Unit, Cipto Mangunkusumo Hospital, was conducted from April 2019 until June 2020. Subjects enrolled in this study were suppressed HIV patients aged between 30-40 years with positive IgG CMV and already using stable ART for at least one year. Transient elastography measured the liver stiffness. Patients with liver stiffness above $7 \mathrm{kPa}$ were defined as having significant liver fibrosis. In addition, Spearman correlation was conducted to evaluate the correlation of clinical presentation of subjects related to liver fibrosis and steatosis.

Results: A total of subjects was included in this study. Dominantly male (62.5\%) with average age $38 \pm 4.68$ years. The median amount of CMV DNA was 466 (17-21284) copy/mL. Significant Fibrosis was found in 17/80 (21\%) subjects. In this study, clinical parameters correlated with liver fibrosis were insulin, glucose fasting, Homa IR, triglyceride, HDL, and platelet. A medium positive correlation was found in insulin, and Homa IR, with coefficient correlation for insulin, was $r=0.475, p<0.001$; and coefficient correlation for Homa IR was $r=0.487, p<0.001$.
\end{abstract}

Conclusion: The prevalence of liver fibrosis was $21 \%$ in these subjects. In addition, insulin and Homa IR had a positive correlation with increasing liver fibrosis.

Keywords: Liver fibrosis, cytomegalovirus, human immunodeficiency virus (HIV) 


\section{ABSTRAK}

Latar belakang: Cytomegalovirus (CMV) adalah virus herpes manusia yang umum terjadi pada pasien human immunodeficiency virus (HIV). Pada pasien dengan imunokompeten, infeksi CMV dapat terjadi asimtomatik jangka panjang yang dapat menyebabkan gangguan fungsi hati dan menimbulkan morbiditas non-AIDS bersama penyakit hati kronik lainnya. Penelitian ini bertujuan untuk mengetahui prevalensi fibrosis dan steatosis hati pada pasien terinfeksi HIV dengan kadar virus sudah ditekan dengan antivirus, namun kondisi infeksi CMV reaktif, serta mengetahui korelasi berbagai manifestasi klinis dengan fibrosis hati pada subjek tersebut.

Metode: Studi potong lintang di Unit Pelayanan Terpadu HIV, Rumah Sakit Cipto Mangunkusumo, dilakukan antara April 2019 hingga Juni 2020. Subyek pada penelitian ini adalah pasien HIV yang kadar virusnya telah ditekan dengan antivirus, berusia antara 30-40 tahun dengan IgG CMV positif dan sudah menggunakan antiretroviral therapy (ART) stabil setidaknya selama satu tahun. Elastografi transien berfungsi mengukur kekakuan hati. Pasien dengan kekakuan hati di atas $7 \mathrm{kPa}$ didefinisikan memiliki fibrosis hati yang signifikan. Selain itu, korelasi Spearman dilakukan untuk mengevaluasi korelasi presentasi klinis subyek penelitian dengan fibrosis dan steatosis hati.

Hasil: Sebanyak 80 pasien dilibatkan dalam penelitian ini. Didominasi laki-laki (62,5\%) dengan usia rata-rata $38 \pm 4,68$ tahun. Jumlah rata-rata DNA CMV adalah 466 (17-21284) copy/mL. Fibrosis signifikan ditemukan pada 17/80 (21\%) pasien. Pada penelitian ini, parameter klinis yang berhubungan dengan fibrosis hati adalah insulin, glukosa puasa, Homa IR, trigliserida, HDL, dan trombosit. Korelasi positif sedang ditemukan pada insulin, dan Homa IR, dengan koefisien korelasi untuk insulin, adalah $r=0,475$, p-value <0,001; dan koefisien korelasi untuk Homa IR adalah $r=0,487, p<0,001$.

Simpulan: Prevalensi fibrosis hati adalah 21\% pada subjek ini. Insulin dan Homa IR memiliki korelasi positif dengan peningkatan fibrosis hati.

Kata kunci: Fibrosis hati, cytomegalovirus, human immunodeficiency virus (HIV)

\section{INTRODUCTION}

After introducing antiretroviral treatment (ART) in 1996, the life expectancy of people living with HIV (PLWH) has increased significantly. Non-AIDS diseases are becoming an increasingly important source of morbidity and mortality among HIV-infected people. ${ }^{1}$ The opportunistic infections and neoplasms AIDS-related as the leading causes of morbidity and mortality have shifted to cardiovascular and liver disease. It is estimated that liver-related diseases account for $13-18 \%$ of all-cause mortality in HIVinfected patients. ${ }^{1,2}$ The development of liver fibrosis represents the ultimate common path for the most clinically relevant liver injury. HIV itself can cause liver damage and consequent liver fibrosis (LF) in several ways. Aside from HIV, viral hepatitis, alcoholic and mainly non-alcoholic liver disease have been linked to liver involvement in PLWHA. Another known cause of hepatotoxicity is ART, which raises clinically significant concerns about LF in long-term treatment. ${ }^{3}$

Antiretroviral therapy (ART) has significantly reduced the morbidity and mortality associated with acquired immunodeficiency syndrome (AIDS). ${ }^{4}$ Among many comorbidities, cardiovascular disease (CVD) has become a particular concern. Among HIV-infected patients, smoking (38\% vs. $18 \%$ ), hypertension ( $21 \%$ vs. $16 \%$ ), diabetes ( $12 \%$ vs. $7 \%$ ), and dyslipidemia $(23 \%$ vs. $18 \%)$ have a higher prevalence. ${ }^{5}$ After HIV infection, a decrease in total cholesterol, HDLcholesterol, and LDL-cholesterol, but an increase in triglycerides is observed in untreated individuals. ${ }^{6}$ Nearly half of patients with long-term ART exposure will experience changes in body composition. Lipid metabolism patients with HIV infection may also experience important changes in body fat composition after exposure to antiretroviral therapy. ${ }^{7}$ Immune activation leads to chronic inflammation, which varies in severity and is observed in untreated HIV patients and patients receiving combination antiretroviral treatment (cART). In patients treated with antiretroviral drugs, pro-inflammatory cytokines were reduced, but they did not fully return to normal. In patients receiving HIV treatment, the activation of the innate immune system and insulin resistance are like those described in obesity and type 2 diabetes (DM2). ${ }^{8,9}$ After the innate immune system is activated, insulinrelated protein signals are converted into transcription post-modification results in reduced insulin action. ${ }^{10,11}$ 
Human cytomegalovirus (CMV) often coexists with HIV and accounts for an excessive proportion of memory $\mathrm{T}$ cell responses. Chronic co-infections such as cytomegalovirus (CMV) may lead to a vicious circle of immune activation and reservoir seeding. ${ }^{12}$ Hijacking cytokine and chemokine signalling, manipulation of cell development pathways, and transactivation of HIV expression by CMV may aggravate the persistence of HIV. This series of circumstances has allowed HIV to build a larger pool of latently infected cells that can hide in dormant states for decades in the face of virus-suppressive antiretroviral therapy (ART) ${ }^{13}$ Cytomegalovirus (CMV) is a human herpesvirus common in people with human immunodeficiency virus (HIV). In patients with immunocompetence, long periodic asymptomatic CMV might affect the development of abnormal liver function and contribute to non-AIDS defining morbidity, including chronic liver disease. In addition, there is accumulated evidence that some infectious agents, such as cytomegalovirus (CMV) and varicella-zoster virus (VZV), may accelerate the course of atherosclerotic disease in HIV-infected patients. ${ }^{14}$ This study aims to know the prevalence of liver fibrosis in virally suppressed HIV infected patients with CMV reactive and summarize the correlation of clinical presentation and liver fibrosis in these subjects.

\section{METHOD}

A Cross-sectional study in HIV Integrated Care Unit, Cipto Mangunkusumo Hospital, was conducted from April 2019 until June 2020. Patients between the ages of 20 and 45, who had used stable ART for at least one year, were CMV IgG positive and had an HIV RNA viral load $<50$ copies/mL, including men and women, were recruited in this study. Patients with the following conditions were excluded from the study: patients undergoing DAA treatment for hepatitis $\mathrm{C}$, decompensated cirrhosis or acute liver failure, history of coronary artery disease, diabetes, brain infection, epilepsy, stroke, rhabdomyolysis or myopathy, severe depression during pregnancy or breastfeeding during the study.

All potential participants received explanations about the purpose and methods of the research and were informed that they had the right to stop or refuse to participate in the research. Viral load, antiCMV antibody and fasted lipid profile were tested before determining eligibility criteria. Participants with CMV seropositivity, undetectable viral load, and Framingham risk score $>10 \%$ LDL $<130$ or Framingham risk score $<10 \% \mathrm{LDL}<160$ continued the study process. Participants were asked to fast for 10 hours before taking $12 \mathrm{~mL}$ of venous blood: for examination of blood glucose, ALT, CMV DNA, soluble CD4 (sCD4), and transient elastography with Controlled Attenuation Parameter (CAP).

Transient elastography (TE) with CAP was done by a trained hepatologist using a Fibro scan (Echosens ${ }^{\circledR}$ ) device. The criteria for successful examination are ten shots and an interquartile range (IQR) for liver stiffness of less than $20 \%$ of the median value. The cut-off value for steatosis diagnosis using CAP measurement of above $238 \mathrm{~dB} / \mathrm{m}$ and the value for fibrosis diagnosis if the transient elastography measurement showed higher than $7.1 \mathrm{kPa} .14$. This study had been approved by Health Research Ethics Committee-Universitas Indonesia and Cipto Mangunkusumo Hospital (HRECFMUI/CMH).

Table 1. Characteristics of study participant

\begin{tabular}{|c|c|}
\hline Variable & Total \\
\hline \multicolumn{2}{|l|}{ Demography } \\
\hline Age, mean \pm SD & $38 \pm 4.68$ \\
\hline \multicolumn{2}{|l|}{ Sex, $n(\%)$} \\
\hline Male & $50(62.5 \%)$ \\
\hline Female & $30(37.5 \%)$ \\
\hline \multicolumn{2}{|l|}{ Body mass index (BMI), $\mathrm{n}(\%)$} \\
\hline Obese & $15(18.8 \%)$ \\
\hline Overweight & $22(27.5 \%)$ \\
\hline Normal & $33(41.3 \%)$ \\
\hline Underweight & $10(12.5 \%)$ \\
\hline \multicolumn{2}{|l|}{$\begin{array}{l}\text { Alcohol consumption less than one month, } \\
\mathrm{n}(\%)\end{array}$} \\
\hline Yes & $6(7.5 \%)$ \\
\hline No & $74(92.5 \%)$ \\
\hline \multicolumn{2}{|l|}{ Laboratory profile } \\
\hline CMV amount, median (range) & $466(17-21284)$ \\
\hline Hemoglobin, mean \pm SD & $13.90 \pm 1.92$ \\
\hline Leukocyte, mean \pm SD & $6.50 \pm 1.86$ \\
\hline Platelet, mean \pm SD & $273 \pm 62$ \\
\hline Total cholesterol, mean \pm SD & $296 \pm 43.16$ \\
\hline $\mathrm{HDL}$, mean $\pm \mathrm{SD}$ & $50 \pm 14.10$ \\
\hline $\mathrm{LDL}$, mean $\pm \mathrm{SD}$ & $119 \pm 35.56$ \\
\hline Trygliceride, median (range) & $112(46-1393)$ \\
\hline Fasting glucose, mean \pm SD & $86 \pm 11.03$ \\
\hline Insulin, median (range) & $7.75(1.7-63.9)$ \\
\hline Homa IR & $1.69(0.3-17.99)$ \\
\hline \multicolumn{2}{|l|}{ HIV diagnosis } \\
\hline \multicolumn{2}{|l|}{ Years of diagnosis, n (\%) } \\
\hline Above five years & $60(75 \%)$ \\
\hline Under-five years & $20(25 \%)$ \\
\hline \multicolumn{2}{|l|}{ HIV stage, $\mathrm{n}(\%)$} \\
\hline NA & $8(10 \%)$ \\
\hline 4 & $3(3.8 \%)$ \\
\hline 3 & $34(42.5 \%)$ \\
\hline 2 & $5(6.3 \%)$ \\
\hline 1 & $30(37.5 \%)$ \\
\hline $\begin{array}{l}\text { CD4 absolute when diagnosed, median } \\
\text { (range) }\end{array}$ & $113(2-623)$ \\
\hline CD4 recent, median (range) & 589 (149-1482) \\
\hline \multicolumn{2}{|l|}{ Years of starting ARV, $\mathrm{n}(\%)$} \\
\hline Above five years & $60(75 \%)$ \\
\hline Under-five years & $20(25 \%)$ \\
\hline \multicolumn{2}{|l|}{ Liver profile } \\
\hline Fibrosis, median (range) & $4.85(2.60-16.30)$ \\
\hline
\end{tabular}


Data were analyzed using software SPSS version 24. The mean and standard deviation were used for normally distributed data, and for abnormally distributed data, the median (minimum-maximum) was used. P-value was calculated using the student t-test for normally distributed data and the Mann-Whitney test for abnormally distributed data. The percentages were used to represent categorical data. The $\chi 2$ test was used to calculate the p-value for categorical data. The baseline characteristics of patients have compared significant and non-significant Fibrosis and steatosis. A multivariate linear regression analysis was conducted to identify the predictors of the liver disease, and Spearman correlation coefficients were obtained to evaluate the correlation of insulin level, fasting blood glucose, body mass index (BMI), HOMA IR, triglyceride, HDL, platelet count of subjects related to liver fibrosis. After entering all variables into the model, the variables that showed the least significant associations were subsequently excluded until all variables remained significant $(p<0.05)$. The model fit for the multiple regression was assessed using the
R2 - coefficient of determination and the adjusted R2 - coefficient of determination, adjusted for the number of independent variables in the model.

\section{RESULTS}

A total of 80 patients was included in this study. Dominantly male (62.5\%) with average age $38 \pm 4.68$ years. The median amount of CMV DNA was 466 (17-21284) copy/mL. Significant fibrosis was found in $17 / 80(21 \%)$ patients, and significant steatosis were 16/80 (20\%) (Table 1). Clinical parameters such as status of smoking, hemoglobin level, triglyceride, insulin, and HOMA-IR, statistically significantly different between significant and non-significant fibrosis and steatosis in bivariate analysis, but not for sex, blood fasting glucose, CD4 absolute when first time diagnosed with HIV, and duration of cART consumption which only statistically significant to differentiate fibrosis group. The level of total cholesterol and LDL seems significant to differentiate the steatosis group (Table 2 and 3 ).

Table 2. Comparison of baseline characteristic of significant and non-significant fibrosis

\begin{tabular}{|c|c|c|c|c|}
\hline & Total & $\begin{array}{l}\text { Significant fibrosis* } \\
(\mathrm{n}=17)\end{array}$ & $\begin{array}{l}\text { Non significant } \\
\text { fibrosis } \\
(n=63)\end{array}$ & $\mathbf{p}$ \\
\hline \multicolumn{5}{|l|}{ Demography } \\
\hline Age, mean $\pm S D$ & $38 \pm 4.68$ & $39 \pm 3.20$ & $38 \pm 4.98$ & 0.285 \\
\hline \multicolumn{5}{|l|}{ Sex, n (\%) } \\
\hline Male & $50(62.5 \%)$ & $16(94.1 \%)$ & $34(54 \%)$ & \multirow[t]{2}{*}{0.002} \\
\hline Female & $30(37.5 \%)$ & $1(5.9 \%)$ & $29(46 \%)$ & \\
\hline \multicolumn{5}{|l|}{ Body mass index (BMI), n (\&) } \\
\hline Obese & $15(18.8 \%)$ & $6(35.3 \%)$ & $9(14.3 \%)$ & \multirow[t]{4}{*}{0.239} \\
\hline Overweight & $22(27.5 \%)$ & $4(23.5 \%)$ & $18(28.6 \%)$ & \\
\hline Normal & $33(41.3 \%)$ & $6(35.3 \%)$ & $27(42.9 \%)$ & \\
\hline Underweight & $10(12.5 \%)$ & $1(5.9 \%)$ & $9(14.3 \%)$ & \\
\hline Systole blood pressure, mean \pm SD & $121 \pm 16.40$ & $129 \pm 24.70$ & $119 \pm 12.70$ & 0.101 \\
\hline Diastole blood pressure, mean \pm SD & $79 \pm 11.20$ & $82 \pm 12.80$ & $78 \pm 10.60$ & 0.138 \\
\hline \multicolumn{5}{|l|}{ Smoker, n (\%) } \\
\hline Current & $22(27.5 \%)$ & $5(29.4 \%)$ & $17(27.0 \%)$ & \multirow[t]{3}{*}{0.017} \\
\hline Former & $15(18.8 \%)$ & $7(41.2 \%)$ & $8(12.7 \%)$ & \\
\hline No & $43(53.8 \%)$ & $5(29.4 \%)$ & $38(60.3 \%)$ & \\
\hline \multicolumn{5}{|c|}{ Alcohol consumption less than one month, $n(\%)$} \\
\hline Yes & $6(7.5 \%)$ & $2(11.8 \%)$ & $4(6.3 \%)$ & \multirow[t]{2}{*}{0.815} \\
\hline No & $74(92.5 \%)$ & $15(88.2 \%)$ & $59(93.7 \%)$ & \\
\hline \multicolumn{5}{|l|}{ Hematology and lipid profile } \\
\hline CMV amount, median (range) & $466(17-21284)$ & $370(172-1471)$ & $480(17-21284)$ & 0.332 \\
\hline Hemoglobin, mean \pm SD & $13.90 \pm 1.92$ & $14.72 \pm 1.36$ & $13.67 \pm 1.99$ & 0.044 \\
\hline Hematokrit, mean \pm SD & $38.81 \pm 5.59$ & $40.26 \pm 3.20$ & $38.42 \pm 6.04$ & 0.097 \\
\hline Leukocyte, mean \pm SD & $6.50 \pm 1.86$ & $6.67 \pm 2.46$ & $6.45 \pm 1.68$ & 0.678 \\
\hline Platelet, mean \pm SD & $273 \pm 62$ & $249 \pm 63$ & $280 \pm 61$ & 0.068 \\
\hline Neutrophil, mean \pm SD & $54.70 \pm 9.82$ & $52.41 \pm 10.14$ & $55.32 \pm 9.73$ & 0.281 \\
\hline Lymphocyte, mean \pm SD & $34.89 \pm 9.34$ & $35.99 \pm 10.04$ & $34.60 \pm 9.20$ & 0.587 \\
\hline Monocyte, mean \pm SD & $7.29 \pm 2.01$ & $8.02 \pm 2.34$ & $7.09 \pm 1.88$ & 0.088 \\
\hline Total cholesterol, mean \pm SD & $296 \pm 43.16$ & $202 \pm 63.13$ & $195 \pm 36.50$ & 0.636 \\
\hline $\mathrm{HDL}$, mean $\pm \mathrm{SD}$ & $50 \pm 14.10$ & $45 \pm 9.66$ & $52 \pm 14.74$ & 0.054 \\
\hline LDL, mean \pm SD & $119 \pm 35.56$ & $112 \pm 49.44$ & $121 \pm 31.07$ & 0.518 \\
\hline Trygliceride, median (range) & $112(46-1393)$ & $173(70-1393)$ & $104(46-332)$ & 0.004 \\
\hline Fasting glucose, mean \pm SD & $86 \pm 11.03$ & $92 \pm 13.09$ & $84 \pm 9.92$ & 0.029 \\
\hline Insulin, median (range) & $7.75(1.7-63.9)$ & $12(7-64)$ & $6.70(2-34)$ & 0.001 \\
\hline Homa IR & $1.69(0.3-17.99)$ & $3.40(1.54-17.99)$ & $1.30(0.30-8.41)$ & 0.001 \\
\hline \multicolumn{5}{|l|}{ HIV diagnosis } \\
\hline \multicolumn{5}{|l|}{ Years of diagnosis, $\mathrm{n}(\%)$} \\
\hline Above 5 years & $60(75 \%)$ & $16(94.1 \%)$ & $44(69.8 \%)$ & \multirow[t]{2}{*}{0.057} \\
\hline Under 5 years & $20(25 \%)$ & $1(5.9 \%)$ & $19(30.2 \%)$ & \\
\hline
\end{tabular}




\begin{tabular}{|c|c|c|c|c|}
\hline & Total & $\begin{array}{l}\text { Significant fibrosis* } \\
(\mathrm{n}=17)\end{array}$ & $\begin{array}{l}\text { Non significant } \\
\text { fibrosis } \\
(\mathrm{n}=63)\end{array}$ & p \\
\hline \multicolumn{5}{|l|}{ HIV stage, $\mathrm{n}(\%)$} \\
\hline NA & $8(10 \%)$ & $3(17.6 \%)$ & $5(7.9 \%)$ & 0.440 \\
\hline 4 & $3(3.8 \%)$ & $0(0 \%)$ & $3(4.8 \%)$ & \\
\hline 3 & $34(42.5 \%)$ & $9(52.9 \%)$ & $25(39.7 \%)$ & \\
\hline 2 & $5(6.3 \%)$ & $1(5.9 \%)$ & $4(6.3 \%)$ & \\
\hline 1 & $30(37.5 \%)$ & $4(23.5 \%)$ & $26(41.3 \%)$ & \\
\hline CD4 absolute when diagnosed, median (range) & $113(2-623)$ & $42(2-573)$ & $143(5-623)$ & 0.011 \\
\hline CD4 recent, median (range) & $589(149-1482)$ & $671(318-1338)$ & $584(149-1482)$ & 0.651 \\
\hline \multicolumn{5}{|l|}{ Years of starting ARV, n (\%) } \\
\hline Above 5 years & $60(75 \%)$ & $16(94.1 \%)$ & $44(69.8 \%)$ & 0.057 \\
\hline Under 5 years & $20(25 \%)$ & $1(5.9 \%)$ & $19(30.2 \%)$ & \\
\hline \multicolumn{5}{|l|}{ Years of NRTI, n (\%) } \\
\hline Above 5 years & $65(81.3 \%)$ & $17(100 \%)$ & $48(76.2 \%)$ & 0.032 \\
\hline Under 5 years & $15(18.8 \%)$ & $0(0 \%)$ & $15(23.8 \%)$ & \\
\hline
\end{tabular}

Significant fibrosis $\geq 7 \mathrm{kPa}$

CMV: Cytomegalovirus, HDL: high density lipoprotein, LDL: low density lipoprotein, HIV: human immunodeficiency virus, ARV: antiretroviral

Table 3. Comparison of baseline characteristic of significant and non-significant steatosis

\begin{tabular}{|c|c|c|c|c|}
\hline & Total & $\begin{array}{l}\text { S1-S3 } \\
\text { significant steatosis } \\
(n=16)\end{array}$ & $\begin{array}{l}\text { So } \\
\text { non-significant } \\
\text { steatosis } \\
(n=64)\end{array}$ & $\mathbf{p}$ \\
\hline \multicolumn{5}{|l|}{ Demography } \\
\hline Age, mean \pm SD & $38 \pm 4.68$ & $38 \pm 3.79$ & $38 \pm 4.90$ & 0.831 \\
\hline \multicolumn{5}{|l|}{ Sex, $n(\%)$} \\
\hline Male & $50(62.5 \%)$ & $10(62.5 \%)$ & $40(62.5 \%)$ & \multirow{2}{*}{1.000} \\
\hline Female & $30(37.5 \%)$ & $6(37.5 \%)$ & $24(37.5 \%)$ & \\
\hline \multicolumn{5}{|l|}{ BMI, n (\&) } \\
\hline Obese & $15(18.8 \%)$ & $6(37.5 \%)$ & $9(14.1 \%)$ & \multirow[t]{4}{*}{0.077} \\
\hline Overweight & $22(27.5 \%)$ & $5(31.3 \%)$ & $17(26.6 \%)$ & \\
\hline Normal & $33(41.3 \%)$ & $5(31.3 \%)$ & $28(43.8 \%)$ & \\
\hline Underweight & $10(12.5 \%)$ & $0(0 \%)$ & $10(15.6 \%)$ & \\
\hline Systole blood pressure, mean \pm SD & $121 \pm 16.40$ & $127 \pm 16.77$ & $119 \pm 16.13$ & 0.121 \\
\hline Diastole blood pressure, mean \pm SD & $79 \pm 11.20$ & $82 \pm 11.78$ & $78 \pm 10.95$ & 0.154 \\
\hline \multicolumn{5}{|l|}{ Smoker, n (\%) } \\
\hline Current & $22(27.5 \%)$ & $3(18.8 \%)$ & $19(29.7 \%)$ & \multirow[t]{3}{*}{0.017} \\
\hline Former & $15(18.8 \%)$ & $7(43.8 \%)$ & $8(12.5 \%)$ & \\
\hline \multirow{2}{*}{\multicolumn{5}{|c|}{ Alcohol consumption, $\mathrm{n}(\%)$}} \\
\hline & & & & \\
\hline Yes & $6(7.5 \%)$ & $1(6.3 \%)$ & $5(7.8 \%)$ & \multirow[t]{2}{*}{1.000} \\
\hline No & $74(92.5 \%)$ & $15(93.8 \%)$ & $59(92.2 \%)$ & \\
\hline \multicolumn{5}{|l|}{ Hematology and lipid profile } \\
\hline CMV amount, median (range) & $466(17-21284)$ & $475(145-1378)$ & $465(17-21284)$ & 0.596 \\
\hline Hemoglobin, mean \pm SD & $13.90 \pm 1.92$ & $14.71 \pm 1.24$ & $13.69 \pm 2.01$ & 0.015 \\
\hline Hematokrit, mean \pm SD & $38.90 \pm 1.92$ & $40.28 \pm 3.39$ & $38.45 \pm 5.98$ & 0.112 \\
\hline Leukocyte, mean \pm SD & $6.50 \pm 1.86$ & $7.15 \pm 2.09$ & $6.34 \pm 1.77$ & 0.119 \\
\hline Platelet, mean \pm SD & $273 \pm 62$ & $288 \pm 72$ & $268 \pm 59.49$ & 0.254 \\
\hline Neutrophil, mean \pm SD & $55 \pm 9.83$ & $57.59 \pm 7.90$ & $53.98 \pm 10.18$ & 0.190 \\
\hline Lymphocyte, mean \pm SD & $34.89 \pm 9.34$ & $32.43 \pm 6.57$ & $35.51 \pm 9.86$ & 0.241 \\
\hline Monocyte, mean \pm SD & $7.29 \pm 2.01$ & $7.03 \pm 2.31$ & $7.35 \pm 1.94$ & 0.566 \\
\hline Total cholesterol, mean \pm SD & $196 \pm 43.16$ & $229 \pm 49.52$ & $188 \pm 37.47$ & 0.001 \\
\hline $\mathrm{HDL}$, mean $\pm \mathrm{SD}$ & $50.95 \pm 14.10$ & $47.31 \pm 16.20$ & $51.86 \pm 13.51$ & 0.251 \\
\hline LDL, mean \pm SD & $119.36 \pm 35.56$ & $139.50 \pm 43.43$ & $114.33 \pm 31.74$ & 0.010 \\
\hline Trygliceride, median (range) & $112(46-1393)$ & 157 (96-1393) & $103(46-448)$ & 0.002 \\
\hline Fasting glucose, mean \pm SD & $86 \pm 11.03$ & $82 \pm 9.66$ & $87 \pm 11.27$ & 0.190 \\
\hline Insulin, median (range) & $7.7 \overline{5}(1.7-63.9)$ & $10.6(5.4-24.4)$ & $7.0(1.7-63.9)$ & 0.001 \\
\hline Homa IR & $1.69(0.3-17.99)$ & $2.19(1.04-5.78)$ & $1.37(0.3-17.99)$ & 0.006 \\
\hline \multicolumn{5}{|l|}{ HIV diagnosis } \\
\hline \multicolumn{5}{|l|}{ Years of diagnosis, $\mathrm{n}(\%)$} \\
\hline Above 5 years & $60(75 \%)$ & $13(81.3 \%)$ & $47(73.4 \%)$ & \multirow[t]{2}{*}{0.749} \\
\hline Under 5 years & $20(25 \%)$ & $3(18.8 \%)$ & $17(26.6 \%)$ & \\
\hline \multicolumn{5}{|l|}{ HIV stage, n (\%) } \\
\hline NA & $8(10 \%)$ & $0(0 \%)$ & $8(12.5 \%)$ & \multirow[t]{5}{*}{0.418} \\
\hline 4 & $3(3.8 \%)$ & $1(6.3 \%)$ & $2(3.1 \%)$ & \\
\hline 3 & $34(42.5 \%)$ & $6(37.5 \%)$ & $28(43.8 \%)$ & \\
\hline 2 & $5(6.3 \%)$ & $2(12.5 \%)$ & $3(4.7 \%)$ & \\
\hline 1 & $30(37.5 \%)$ & $7(43.8 \%)$ & $23(35.9 \%)$ & \\
\hline CD4 absolute when diagnosed, median (range) & $113(2-623)$ & $71(2-579)$ & $135(11-623)$ & 0.061 \\
\hline CD4 recent, median (range) & $589(149-1482)$ & $685(268-1338)$ & $576(149-1482)$ & 0.178 \\
\hline \multicolumn{5}{|l|}{ Years of starting ARV, $\mathrm{n}(\%)$} \\
\hline Above five years & $60(75 \%)$ & $12(75 \%)$ & $48(75 \%)$ & \multirow[t]{2}{*}{1.000} \\
\hline Under 5 years & $20(25 \%)$ & $4(25 \%)$ & $16(25 \%)$ & \\
\hline \multicolumn{5}{|l|}{ Years of NRTI, n (\%) } \\
\hline Above 5 years & $15(18.8 \%)$ & $13(81.3 \%)$ & $52(81.3 \%)$ & \multirow[t]{2}{*}{1.000} \\
\hline Under 5 years & $60(75 \%)$ & $3(18.8 \%)$ & $12(18.8 \%)$ & \\
\hline
\end{tabular}

** S0 $<248 \mathrm{db} / \mathrm{m}, \mathrm{S} 1=248-268 \mathrm{db} / \mathrm{m}, \mathrm{S} 2=268-280 \mathrm{db} / \mathrm{m}, \mathrm{S} 3>280 \mathrm{db} / \mathrm{m}$

CMV: Cytomegalovirus, HDL: high density lipoprotein, LDL: Iow density lipoprotein, HIV: human immunodeficiency virus, ARV: antiretroviral 
The clinical parameters which significantly have positive moderate correlation with liver fibrosis were insulin and HOMA-IR ( $\mathrm{r}=0.475 ; \mathrm{p}<0.001$ vs. $\mathrm{r}=$ 0.487; $\mathrm{p}<0.001$ ), whereas blood glucose fasting, and triglyceride level showed significant positive low correlation with liver fibrosis $(\mathrm{r}=0.247 ; \mathrm{p}=$ 0.027 vs. $r=0.241 ; p=0.031)$. The HDL level has significant negative low correlation with liver fibrosis and steatosis $(\mathrm{r}=-0.235 ; \mathrm{p}=0.036$ vs. $\mathrm{r}=-0.288 ; \mathrm{p}=$ 0.010) (Table 4).

Table 4. Correlation of fibrosis and steatosis with clinical parameters

\begin{tabular}{lllll}
\hline $\begin{array}{l}\text { Independent } \\
\text { Variable }\end{array}$ & $\begin{array}{l}\text { CCoefficient } \\
\text { Ccorrelation } \\
\text { of Ffibrosis } \\
(\mathbf{r})\end{array}$ & P-Value & \multicolumn{3}{l}{$\begin{array}{l}\text { Coefficien } \\
\text { Correlation } \\
\text { of } \\
\text { Steatosis } \\
(\mathbf{r})\end{array}$} & $\mathbf{P}$ *** \\
\hline Insulin & 0.475 & $<0.001$ & 0.374 & 0.001 \\
Glucose Fasting & 0.247 & 0.027 & - & - \\
$\begin{array}{l}\text { Body Mass } \\
\text { Index (BMI) }\end{array}$ & - & - & 0.403 & 0.001 \\
HOMA-IR & 0.487 & $<0.001$ & 0.311 & 0.005 \\
Trygliceride & 0.241 & 0.031 & 0.308 & 0.005 \\
$\begin{array}{l}\text { (High density } \\
\text { lipoprotein) }\end{array}$ & -0.235 & 0.036 & -0.288 & 0.010 \\
HDL & -0.253 & 0.024 & - & - \\
Platelet & & & & \\
\hline *** Statistically significant $\mathrm{p}<0.005$ & & &
\end{tabular}

The clinical parameters which significantly have positive moderate correlation with liver steatosis was only body mass index (BMI) $(r=0.403 ; \mathrm{p}=0.001)$, whereas insulin, HOMA-IR, and triglyceride level showed only significant positive low correlation with liver steatosis $(\mathrm{r}=0.374 ; \mathrm{p}=0.001$ vs. $\mathrm{r}=0.311 ; \mathrm{p}=$ 0.005 vs. $r=0.308 ; p=0.005$ ). (Table 4 )

Multivariate linear regression showed that the following factors, sex, HOMA-IR, and platelet count, could predict the liver fibrosis value $(\mathrm{kPa})$, whereas $\mathrm{BMI}$ and triglyceride could predict the liver steatosis value $(\mathrm{dB} / \mathrm{m})$.

Table 5. Multivariate linear regression related to liver fibrosis

\begin{tabular}{lllll}
\hline $\begin{array}{l}\text { Independent } \\
\text { variable }\end{array}$ & $\begin{array}{l}\text { Regression } \\
\text { coefficient }\end{array}$ & $\begin{array}{l}\text { Standard } \\
\text { error }\end{array}$ & t value & $\mathbf{p}$ \\
\hline Sex & 1.19 & 0.594 & 1.995 & 0.050 \\
HOMA-IR & 0.255 & 0.120 & 2.130 & 0.036 \\
Platelet & -0.010 & 0.005 & -2.270 & 0.026 \\
\hline
\end{tabular}

Prediction Linier Regression Model $\rightarrow Y=\beta 0+\beta_{1} x_{1}+\beta_{2} x_{2}+\beta_{3} x_{3}$ $Y($ Liver Fibrosis $)=7.14+1.19$ (Sex) +0.26 (HOMA-IR) -0.01 (Platelet)

Table 6. Multivariate linear regression related to liver steatosis

\begin{tabular}{lllll}
\hline $\begin{array}{l}\text { Independent } \\
\text { variable }\end{array}$ & $\begin{array}{l}\text { Regression } \\
\text { coefficient }\end{array}$ & $\begin{array}{l}\text { Standard } \\
\text { error }\end{array}$ & t value & $\mathbf{p}$ \\
\hline $\begin{array}{l}\text { Body mass } \\
\text { index (BMI) }\end{array}$ & 4.718 & 1.389 & 3.397 & 0.001 \\
Triglyceride & 0.075 & 0.029 & 2.636 & 0.010 \\
\hline
\end{tabular}

Prediction Linier Regression Model $\rightarrow Y=\beta 0+\beta_{1} x_{1}+\beta_{2} x_{2}+\beta_{3} x_{3}$ $Y($ Liver Steatosis $)=89.28+4.72(B M I)+0.075$ (Triglyceride)

\section{DISCUSSION}

This study highlighted the burden of liver fibrosis and steatosis as assessed by TE in patients with HIV mono-infection under condition viral suppressed with long-term ART. Extensive variability remains regarding the prevalence of liver fibrosis and steatosis in patients with HIV mono-infection. In a study of 62 patients with HIV mono-infection during liver biopsy where transaminase levels continued to rise, Morse et al reported the prevalence of steatosis and bridging fibrosis were as high as $70 \%$ and $18 \%$, respectively. ${ }^{14}$ Lombardi et al followed a limited sample size $(\mathrm{n}=125)$ of patients with persistent HIV infection in European outpatient clinics. The prevalence of steatosis described using abdominal ultrasound and liver stiffness measurement (LSM) $(\geq 7.4 \mathrm{kPa})$ was $55 \%$, and the prevalence of fibrosis was $18 \% .^{14}$

Recently, a large Canadian cohort $(n=541)$ reported a similar prevalence of steatosis $(36 \%)$ using CAP $(\geq 248 \mathrm{~dB} / \mathrm{m})$ and higher fibrosis rates (19\%) using $\operatorname{LSM}(\geq 7.2 \mathrm{kPa})$ in HIV mono-infection. ${ }^{15}$ It is almost similar to our data reported the prevalence of fibrosis and steatosis $21 \%$ and $20 \%$, respectively. Among HIVinfected people, central obesity and the duration of HIV infection have been described by previous studies as critical features of liver fibrosis. ${ }^{15-17}$

In the present study, the duration of ART consumption showed a significant difference between significant and non-significant fibrosis but did not differentiate the degree of steatosis. In several studies, cART-induced hepatotoxicity and other risk factors, which might be causal for the development of liver fibrosis in HIVinfected patients, are controversially discussed. ${ }^{18}$ While on the one hand, effective control of HIV was associated with slower liver fibrosis progression in HIV/HCV co-infected patients, primarily through the use of Protease Inhibitors (PIs), exposure to Didanosine (ddI), on the other hand, has been associated with liver injury. ${ }^{18}$

The higher prevalence of HIV-suppressed steatosis may be related to the weight gain after the initiation of ART and detectable non-ART compliance in HIV patients, enhancing the potential of antiretroviral drugs for the development of liver steatosis effect. Our study showed that the BMI has a moderate correlation with liver steatosis and, even together with triglyceride, could predict the liver steatosis value $(\mathrm{dB} / \mathrm{m})$ based on the multivariate linear regression.

In our study, the triglyceride, LDL, total cholesterol, and HDL levels differed significantly between significant and non-significant steatosis. Some mechanisms could 
contribute to the event of dyslipidemia in HIV. Besides activation of the innate immune system and insulin resistance, which contributes to glucose metabolism dysregulation and dyslipidemia, other mechanisms may also explain this dyslipidemia pattern. Some studies suggest that in HIV-infected patients not undergoing cART, there is an increase in fatty acid production that can contribute to the appearance of dyslipidemia and insulin resistance. On the other side, it has been shown that cART not only suppresses HIV infection and reduces inflammation, but it also changes the dyslipidemia pattern, characterized by an increase in TGs and LDL-C, a reduction in HDL-C and maintenance of insulin resistance. ${ }^{19,20}$

So far, the molecular mechanism of the increased severity of CMV-related liver diseases and the viral protein group related to this process are still unclear. Most current studies are listed under the observation category. However, previous reports on the pathogenicity of CMV in other tissues introduced some CMV proteins as inducers of the fibrotic process. Transfection of renal epithelial cells with plasmids encoding human CMV IE1 or IE2 gene products shows their possible role in the process of fibrosis. The latter conclusion can be demonstrated by the potency of the IE1 and IE2 gene products in inducing TGF- $\beta 1$ activation (a well-known potent fibrotic molecule) and the subsequent potency of the fibrotic phenotype obtained by transfected cells. More importantly, several CMV proteins regulate the mechanism of cell apoptosis. More importantly, several CMV proteins regulate the mechanism of apoptosis. ${ }^{21,22}$

\section{CONCLUSION}

Non-AIDS diseases are becoming an increasingly important source of morbidity and mortality among HIV-infected people. HIV itself can cause liver damage and consequent liver fibrosis in several ways. Lipid metabolism patients with HIV infection may also experience essential changes in body fat composition after exposure to antiretroviral therapy. Cytomegalovirus (CMV) is a human herpes virus common in people with human immunodeficiency virus (HIV). In patients with immunocompetence, long periodic asymptomatic CMV might affect the development of an abnormal liver function.

The HDL level has a significant negative low correlation with liver fibrosis and steatosis in patients with HIV mono-infection. Sex, HOMA-IR, and platelet count were the only factors predicting the liver steatosis value $(\mathrm{dB} / \mathrm{m})$. The prevalence of HIV-suppressed steatosis may be related to the weight gain after the initiation of ART and detectable non-ART compliance in HIV patients.

Some mechanisms could contribute to the event of dyslipidemia in HIV. It has been shown that cART not only suppresses HIV infection and reduces inflammation but it also changes the dyslipidemia pattern.

\section{REFERENCES}

1. Palella FJ, Delaney KM, Moorman AC, Loveless MO, Fuhrer J, Satten GA, et al. Declining Morbidity and Mortality among Patients with Advanced Human Immunodeficiency Virus Infection. N Engl J Med 1998;338:853-60.

2. Smith C. Factors associated with specific causes of death amongst HIV-positive individuals in the D: A:D study: The data collection on adverse events of anti-HIV drugs (D: A:D) study group. Aids 2010;24:1537-48.

3. Bakasis AD, Androutsakos T. Liver fibrosis during antiretroviral treatment in HIV-infected individuals. Truth or tale? Cells 2021;10:5.

4. Wilson PWF, D’Agostino RB, Levy D, Belanger AM, Silbershatz H, Kannel WB. Prediction of coronary heart disease using risk factor categories. Circulation 1998;97:1837-47.

5. Triant VA, Lee H, Hadigan C, Grinspoon SK. Increased acute myocardial infarction rates and cardiovascular risk factors among patients with human immunodeficiency virus disease. J Clin Endocrinol Metab 2007;92:2506-12.

6. Worm SW, Kamara DA, Reiss P, Kirk O, El-Sadr W, Fux C, et al. Elevated triglycerides and risk of myocardial infarction in HIV-positive persons. Aids 2011;25:1497-504.

7. Stanley TL, Grinspoon SK. Body composition and metabolic changes in HIV-infected patients. J Infect Dis 2012;205:S383-90.

8. Monroe AK, Glesby MJ, Brown TT. Diagnosing and managing diabetes in HIV-infected patients: Current concepts. Clin Infect Dis 2015;60:453-62.

9. Duncan AD, Goff LM, Peters BS. Type 2 diabetes prevalence and its risk factors in HIV: A cross-sectional study. PLoS One 2018;13:e0194199.

10. Carvalho-Filho MA, Carvalho BM, Oliveira AG, Guadagnini D, Ueno M, Dias MM, et al. Double-stranded RNA-activated protein kinase is a key modulator of insulin sensitivity in physiological conditions and in obesity in mice. Endocrinology 2012;153:5261-74.

11. Carvalho BM, Oliveira AG, Ueno M, Araújo TG, Guadagnini D, Carvalho-Filho MA, et al. Modulation of double-stranded RNA-activated protein kinase in insulin sensitive tissues of obese humans. Obesity 2013;21:2452-7.

12. Brenchley JM, Schacker TW, Ruff LE, Price DA, Taylor JH, Beilman GJ, et al. CD4+ T cell depletion during all stages of HIV disease occurs predominantly in the gastrointestinal tract. J Exp Med 2004;200:749-59.

13. Siliciano JM, Siliciano RF. The remarkable stability of the latent reservoir for HIV-1 in resting memory CD4+ T cells. J Infect Dis 2015.p.1345-7.

14. Cravens DW, Ingram TN, LaForge RW, Young CE. BehaviorBased and Outcome-Based Salesforce Control Systems. J Mark 1993;57:47. 
15. Pembroke T, Deschenes M, Lebouché B, Benmassaoud A, Sewitch M, Ghali P, et al. Hepatic steatosis progresses faster in HIV mono-infected than HIV/HCV co-infected patients and is associated with liver fibrosis. J Hepatol 2017;67:801-8.

16. Mohr R, Schierwagen R, Schwarze-Zander C, Boesecke C, Wasmuth JC, Trebicka J, et al. Liver fibrosis in HIV patients receiving a modern cART: Which factors play a role? Med (United States) 2015;94:e2127.

17. Lui G, Wong VWS, Wong GLH, Chu WCW, Wong CK, Yung IMH, et al. Liver fibrosis and fatty liver in Asian HIV-infected patients. Aliment Pharmacol Ther 2016;44:411-21.

18. Anadol E, Lust K, Boesecke C, Schwarze-Zander C, Mohr $\mathrm{R}$, Wasmuth JC, et al. exposure to the previous cART is associated with significant liver fibrosis and cirrhosis in human immunodeficiency virus-infected patients. PLoS One 2018;13:e0191118.

19. Haugaard SB, Andersen O, Pedersen SB, Dela F, Fenger $\mathrm{M}$, Richelsen B, et al. Tumor necrosis factor $\alpha$ is associated with insulin-mediated suppression of free fatty acids and net lipid oxidation in HIV-infected patients with lipodystrophy. Metabolism 2006;55:175-82.

20. Shinohara E, Yamashita S, Kihara S, Hirano KI, Ishigami $\mathrm{M}$, Arai T, et al. Interferon-alpha induces lipid metabolism disorder by lowering post heparin lipases and cholesteryl ester transfer protein activities in patients with chronic hepatitis C. Hepatology 1997;25:1502-6.

21. Shimamura M, Murphy-Ullrich JE, Britt WJ. Human cytomegalovirus induces TGF- $\beta 1$ activation in renal tubular epithelial cells after the epithelial-to-mesenchymal transition. PLoS Pathog 2010;6:e1001170.

22. Jeong W Il, Park O, Radaeva S, Gao B. STAT1 inhibits liver fibrosis in mice by inhibiting stellate cell proliferation and stimulating NK cell cytotoxicity. Hepatology 2006;44:1441-51. 